\title{
Application of Powder X - Ray Diffraction in the Identification of Chinese Patent Medicine
}

\author{
Yingxue $\mathrm{Li}^{1, \mathrm{a}}$, Yankun $\mathrm{Li}^{1, \mathrm{~b}}$ and Leilei Zhao ${ }^{2, \mathrm{c}}$ \\ ${ }^{1}$ School of Environmental Science and Engineering Department, North China Electric Power \\ University, Baoding, China, 071003; \\ ${ }^{2}$ School of Energy Power and Mechanical Engineering, North China Electric Power University, \\ Baoding, China, 071003; \\ a602497168@qq.com, b309267061@qq.com, c2773754282@qq.com
}

Keywords: X-ray diffraction method; Identification of Chinese patent medicine; Fourier map; Ginkgo tablet; Bezoar antidotal tablet

Abstract. Powder X-ray diffraction technique is a new scientific method to analyze the structure of the material, which has been applied to a variety of disciplines, such as chemistry, physics, etc. Due to the special properties of the X-ray diffraction Fourier map of each Chinese patent medicine, the geometric topology graph has the characteristic of fingerprint, and there are big differences between two peak and diffraction peak intensity. Therefore, according to the Fourier map, researchers can compare the different manufacturers and the different batches of Chinese patent medicine, to analyze whether the composition and configuration are consistent. This paper verifies the feasibility of the X-ray diffraction method for the identification of Chinese patent medicine by the above comparison, and find out the characteristic marked peaks of the Ginkgo Biloba Leaves Extract Tablets and Niuhuangjiedu tablets (pills).

\section{粉末X射线衍射在中成药鉴定中的应用 李颗雪 ${ }^{1, a}$, 李艳坤 ${ }^{1, b}$, 赵蕾蕾 ${ }^{2, c}$}

1. 华北电力大学(保定) 环境科学与工程学院 中国 河北保定 071003

2. 华北电力大学(保定) 能源动力与机械工程学院 机械工程系 中国 河北 保定 071003 a602497168@qq.com, b309267061@qq.com, c2773754282@qq.com

摘要: 粉末 X 射线衍射技术是一种分析物质结构的新型科学方法, 目前已经应用到化学、物 理等多种学科。由于每种中成药的 X 射线衍射 Fourier 图谱所具有的几何拓扑图形具有指纹特 征的专属性, 两两之间的峰形和衍射峰强度存在较大的差异, 因此根据得到的 Fourier 图谱, 可对比分析出不同厂家、不同批次的中成药中的成分、晶型结构是否一致。本文通过上述对 比验证了 X 射线衍射法运用于中成药鉴定可行性，并找出了银杏叶片、牛黄解毒片（丸）的 特征标记峰。

关键词：X 射线衍射法; 中成药鉴定；Fourier 图谱; 银杏叶片；牛黄解毒片;

\section{1. 引言}

中成药的历史由来已久, 其成分复杂, 常常不能以简单的化学式来表达。而不同厂家不同批 次生产的中成药的成分也有细微的差距。正是由于中成药的配方多样, 有效成分不明, 给中 成药的鉴定带来困难。用 X 射线衍射法鉴定中药也是近年来才出现的新方法, 它的操作简单, 且测量精度很高, 已经得以广泛推广。它是依据物质在经 $X$ 射线照射后, 由于其物质组成、 分子键、分子构型等的差异会产生其独有的衍射图谱来鉴定的，不会损伤物质，且具有高效 
无污染、测量精度高的特点。

\section{2. 实验条件}

采用丹东浩元仪器公司生产的 DX-2800 高分辨 X 射线衍射仪进行实验, 并记录数据, 使用 Jade 5 分析数据。

样品制备：所有的药片都去糖衣、胶囊去外壳后研磨成粉末，过 100 目篮，制成 X 射线衍射 的粉末样品, 蜜丸剂则直接压制成光滑的片状进行实验。

\section{3. 不同厂家生产的中成药 X 射线衍射图谱}

3. 1. 不同生产厂家的银杏叶片的 Fourier 图谱

银杏叶片具有活血化瘀的功效, 主治冠心病稳定型心绞痛等症状, 主要成分为银杏叶提取物, 其成分复杂, 化学成分主要为黄酮苷类、萜内酯类、聚异戊烯醇类、有机酸、银杏酚酸类等。 先对五种不同厂家生产的银杏叶片进行 X 射线衍射分析, 扫描条件均为: 步宽 0.05 , 角度 $4^{\circ}$ $-64^{\circ}$, 采样时间 1 , 扫描速度 $3^{\circ} / \mathrm{min}$ 。

表 1 不同厂家生产的银杏叶片及编号

\begin{tabular}{cccccc}
\hline 编号 & $1-1$ & $1-2$ & $1-3$ & $1-4$ & $1-5$ \\
\hline \multirow{2}{*}{ 厂家 } & 广西金嗓子 & 贵州信邦 & 德国威玛舒培 & 山东省烟台荣 & 深圳海王 \\
& 药业 & 制药 & 博士药厂 & 昌制药 & 药业 \\
\hline
\end{tabular}

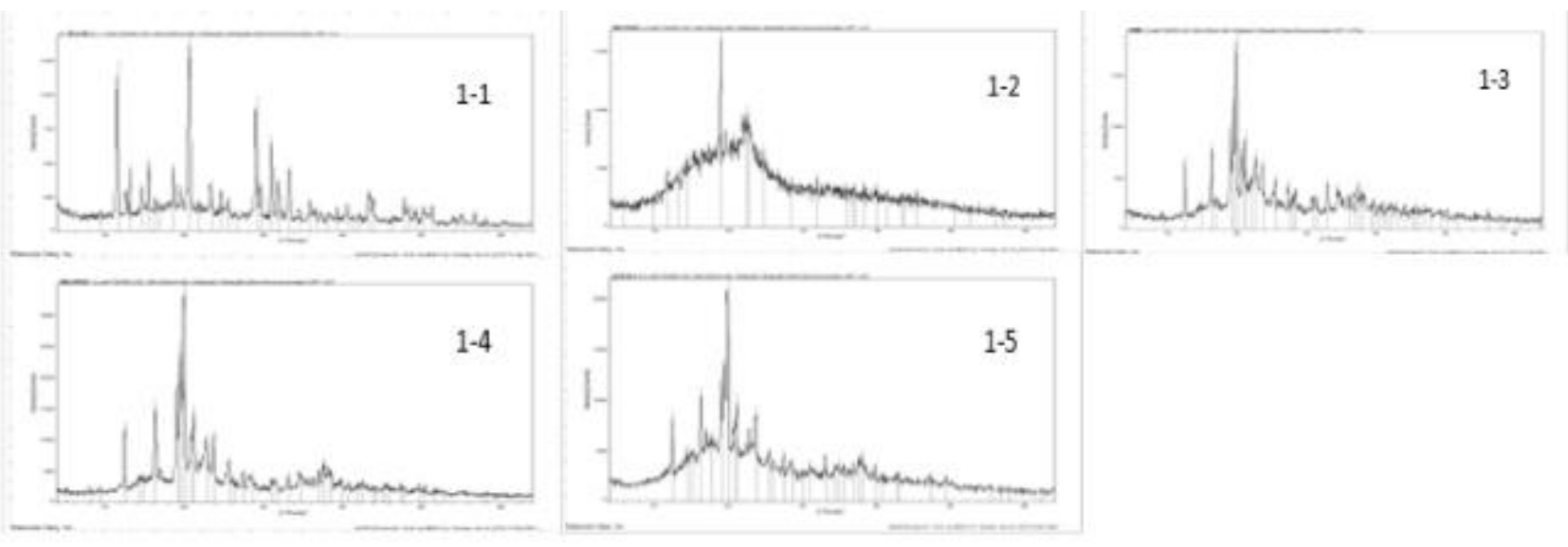

图 1

从图 1 中可以看出，1-3、1-4、1-5 所代表的厂家生产的银杏叶片的 X 射线衍射 Fourier 图 谱的几何拓扑规律基本一致，1-1、1-2 则与它们稍有差别。1-3、1-4、1-5 的衍射图谱共同 具有的 18 个衍射峰的均值为: $12.557 / 621,16.437 / 708,19.148 / 922,19.849 / 1537$, 21. 247/595, 23.752/397, 25.631/232, 27.299/195, 28.541/164, 33.166/209, 34.639/149, 37. 551/173，39.832/128，40.623/78，42.490/88，47.278/79，49.406/80，57.734/43，可 成为银杏叶片的特征标记峰。

图中 1-1 代表广西金嗓子药业所生产的，图谱衍射峰共有 40个; 1-2 代表贵州信邦制药所生 产的, 图谱衍射峰共有 24 个, 该图谱的峰形较为弥散, 推测为非晶态物质形成的弥散峰形。 而 1-1 图谱则与 1-3、1-4、1-5 图谱一样，是由晶态的锐锋和非晶态的弥散峰共同构成，然 而峰形上有所差异可能是由于原材料银杏叶的不同而造成的。由图中对比可以看出, 1-1 图 谱比 1-3、1-4、1-5 图谱的高峰多两组, 因此可推测广西金嗓子药业生产的银杏叶片中可能 比其他厂家生产的药片组分数更多。

造成 1-2 图谱主要形成非晶态弥散峰形的原因推测为加工工艺不同导致物质结晶程度不同, 贵州信邦生产的药片主要为非晶态, 可能是由于生产过程中冷却药片成型的速度过快造成的。 3.2. 不同厂家生产的牛黄解毒片及牛黄解毒丸的 Fourier 图谱 
牛黄解毒片（丸）的主要成分为人工牛黄、雄黄、石膏、大晃、黄芩、桔梗、冰片、甘草, 具有清热解毒的功效。市场上该种药物可分为片剂和丸剂, 片剂多糖衣包覆, 本节中探究了 不同厂家生产的片剂成分, 以及相同厂家生产的片剂和丸剂的区别。

制样过程均去糖衣，大蜜丸压制成薄片，使得表面平滑。

扫描条件均为: 步宽 0.05 , 角度 $3^{\circ}-63^{\circ}$, 采样时间 1 , 扫描速度 $3^{\circ} / \mathrm{min}$ 。

表 2 不同厂家生产的牛黄解毒片 (丸) 及编号

\begin{tabular}{l|ll|l|l|l}
\hline & \multicolumn{3}{|c|}{ 牛黄解毒片 } & \multicolumn{3}{c}{ 牛黄解毒丸 } \\
\hline 编号 & $2-1$ & $2-2$ & $2-3$ & $2-4$ & $2-5$ \\
\hline 厂家 & 昆明中药厂 & 郑州瑞龙制药 & 北京同仁堂科技发展制药厂 & $\begin{array}{l}\text { 吉林吉春 } \\
\text { 制药公司 }\end{array}$ \\
\hline
\end{tabular}
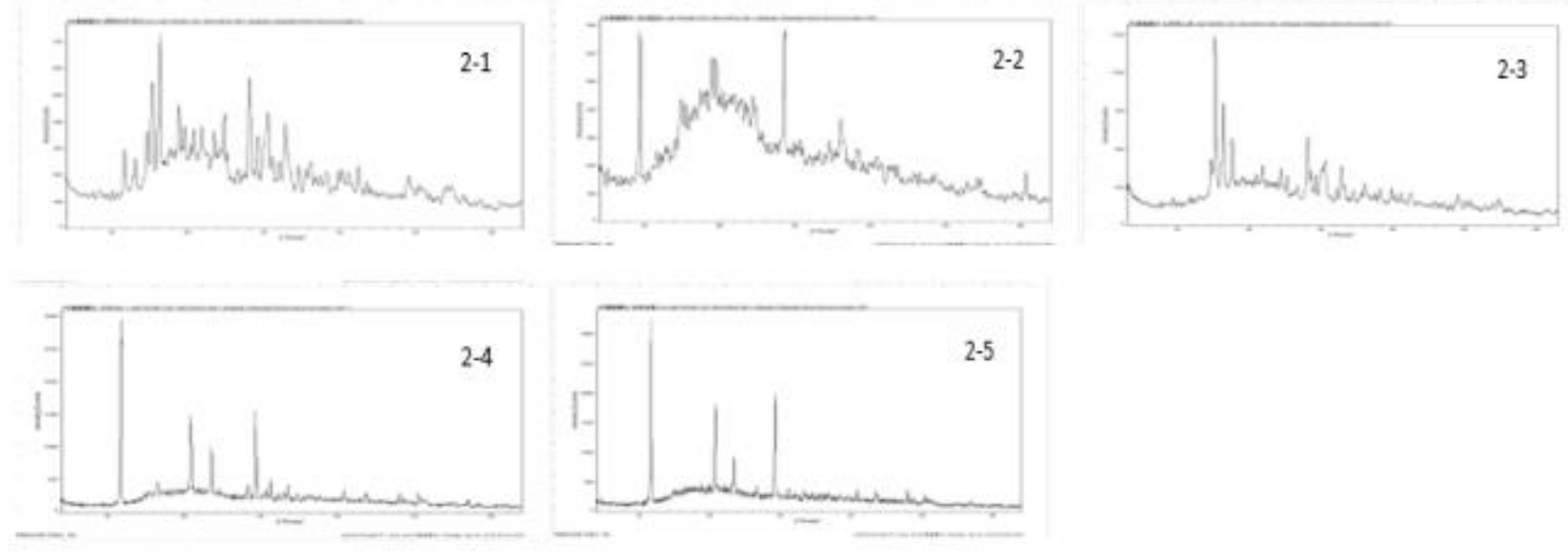

图 2

图 2 中, 2-1 至 2-3 为牛黄解毒片的 X 射线衍射 Fourier 图谱, 2-4 和 2-5 为牛黄解毒丸的 X 射线衍射 Fourier 图谱。从图中可以较为明显地看出, 2-2 是非晶态的弥散峰形, 2-3 为晶态的 锐锋和非晶态的弥散峰叠加, 2-1 则介于两者之间。这三者的图谱几何拓扑规律较为一致, 其 共同具有的 9 个衍射峰的均值为: $14.837 / 177,15.431 / 476,24.604 / 148,28.492 / 319$, $30.619 / 183 ， 34.616 / 78 ， 36.129 / 116 ， 38.278 / 69 ， 42.507 / 74$, 可成为牛黄解毒片的特征标 记峰。由于牛黄解毒片的组分较为丰富, 不同生产厂家的制药配比有所差异, 且由制造工艺 的不同而引起晶态的含量不同 (初步可判断北京同仁堂科技发展制药厂生产的牛黄解毒片含 晶态物质最多), 故造成共同具有的衍射峰个数不多。

图中 2-4 和 2-5 所得图谱的几何拓扑规律几乎完全一致, 其共同具有的 14 个衍射峰的均值为: 11. 838/2959, 15. 505/81, 20. 945/1317, 23. 582/636, 29. 325/1525, 31.307/226, 33. 562/161, $36.810 / 85$, 40.830/175, 43.576/152, 48.567/77, 50.525/136, 57.428/69, 58.423/43, 可成为牛黄解毒丸的特征标记峰。

对比 2-3 和 2-4 可得, 同一厂家生产的片剂和丸剂中, 丸剂所含有的晶态物质较多。调查片剂 和丸剂的生产过程可知, 生产丸剂有较为明确的组方规定, 如牛黄 (5g)、雄黄 (50g)、冰片

(25g)、甘草 (50g) 等粉碎制为细粉, 后加蜜制成蜜丸。而片剂的生产则较为复杂, 经过混 合、滤渣成亳、干燥压制等工序, 因此也可以解释不同厂家生产的片剂的 Fourier 图谱差异较 大，而丸剂谱图基本一致。

\section{4. 不同批次生产的中成药 X 射线衍射图谱}

4.1. 不同批次生产的牛黄解毒丸的 Fourier 图谱 
第二节中分析了不同厂家生产的牛黄解毒片及牛黄解毒丸的 Fourier 图谱, 由于牛黄解毒丸图 谱的峰高明显, 弥散峰形低, 图谱明确易分辨, 进而研究了同一厂家 (北京同仁堂科技发展 制药厂）两个批次生产的牛黄解毒丸的 Fourier 图谱。

\section{表 3 不同生产批次的牛黄解毒丸及编号}

\begin{tabular}{c|lll}
\hline 生产厂家 & 编号 & $3-1$ & $3-2$ \\
\hline 北京同仁堂科技发展制药厂 & 批次 & 692 & 679
\end{tabular}

从图 3 中可以看出, 两个批次的牛黄解毒丸所得到的 Fourier 图谱几乎完全一致, 可得到其特 征峰的均值作为鉴定牛黄解毒丸的特征标记峰： 19.230/88，24.383/373，28.501/210， 29.495/1185, 31.209/150, 33.462/148, 36.714/99, 41.254/100, 46.731/48, 48.509/127, 50. 479/48。

同一厂家生产工艺和组分配方相同, 因而得到的晶态的成分也相差甚微, 从该鉴定结果看来, 批次不同的牛黄解毒丸可以得到几乎完全相同的 Fourier 图谱, 并可取其均值作为牛黄解毒丸 的特征标记峰。
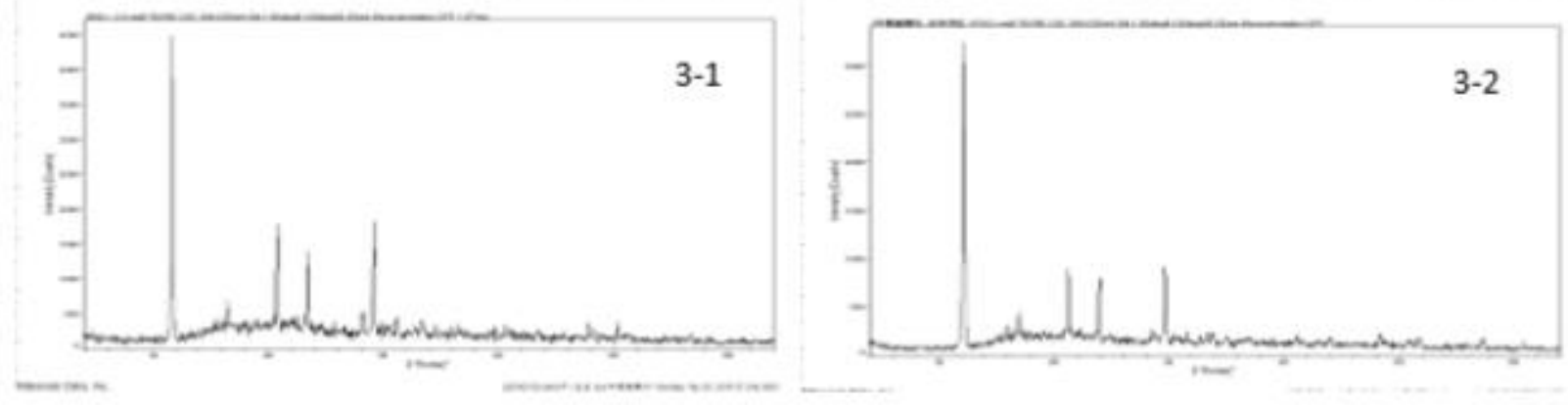

\section{5. 结论}

图 2

本文通过对比分析不同生产厂家的银杏叶片、牛黄解毒片, 同一厂家生产的牛黄解毒片剂和 丸剂, 同一厂家生产的不同批次的牛黄解毒丸, 得出相应中成药的特征标记峰, 可以作为该 中成药的鉴定峰。并根据结果可以看出, 不同生产厂家由于制造工艺和组分配方不同, 生产 的药物中晶态和非晶态的含量不同, 当制造工艺和配方有严格规定时, 即使是不同厂家生产 的药物所得的 Fourier 图谱也基本完全一致。若能得到大量药物的衍射图谱, 可以根据统计学、 化学计量学的方法初步判别某一未知产地未知名称的药物的产地及类型, 从而实现中成药的 鉴定。

\section{References}

[1] B. Zhao: Single Crystal Structure Analysis for Natural Compounds and Identification of Traditional Chinese Medicine by Powder X-ray Diffraction Fourier Pattern. (ph.D., Peking Union Medical College, China 2002). p.202-217.

[2] J.H. Yang: Methodology Research on XRD Second Derivative Fingerprint of Traditional Chinese Medicine (MS. Sichuan University, China 2006). p.18-20.

[3] W.W. Dong and X.P. Liu: China Medical Herald, Vol. 4(2007) No. 18, p.186.

[4] X.P. Liu, X.Y. Chen and Q. Song: Chinese Herbal Medicines, Vol. 28(2005) No. 3, p.184

[5] Z.H. Tian, X.H. Zhang and Z.G. Tian: Engineering \& Test, Vol. 49(2009) No.3, p.40 
[6] Safa Kasap, Joel B. Frey and George Belev: Amorphous and Polycrystalline Photoconductors for Direct Conversion Flat Panel X-Ray Image Sensors, Sensors (Basel) Vol. 11(2011) No. 5, p. 5112. Published online 2011 May 9.

[7] Hiroshige Mori, Kichiro Koshida and Osamu Ishigamori: Evaluation of the effectiveness of X-ray protective aprons in experimental and practical fields, Radiol Phys Technol. Vol. 7(2014) No. 1, p. 158. Published online 2013 December 13.

[8] Di Wu, Aimin Yan and Molly D. Wong: Characterization of a high-energy in-line phase contrast tomosynthesis prototype, Med Phys. Vol. 42(2015) No. 5, p. 2404. Published online 2015 April 17.

[9] Nivedh Manohar, Bernard L. Jones and Sang Hyun Cho: Improving x-ray fluorescence signal for benchtop polychromatic cone-beam x-ray fluorescence computed tomography by incident x-ray spectrum optimization: A Monte Carlo study, Med Phys. Vol. 41(2014) No. 10, p.106. Published online 2014 September 17.

[10] Jinwoo Choi, Perry Radau, Robert Xu and Graham A. Wright: X-ray and magnetic resonance imaging fusion for cardiac resynchronization therapy, Medical Image Analysis. Vol. 31(2016), p. $98-107$ 